\title{
Relationship between obesity and serum reactive oxygen metabolites in adolescents
}

\author{
Terumi Kogawa $\cdot$ Ikuo Kashiwakura
}

Received: 12 October 2012/Accepted: 21 April 2013/Published online: 10 May 2013

(C) The Japanese Society for Hygiene 2013

\begin{abstract}
Objectives Various cross-sectional studies have revealed a significant positive relationship between systemic oxidative stress and obesity-related indices such as body mass index (BMI, $\left.\mathrm{kg} / \mathrm{m}^{2}\right)$. However, little is known of the role of oxidative stress during adolescence. The aim of this study was to determine the association between obesity and serum reactive oxygen metabolites (ROM) in adolescents. Method A total of 595 healthy junior high school students from northern Japan were enrolled in the study. Oxidative stress was evaluated by measuring serum levels of ROM. Obesity indices included BMI and percentage body fat (PBF). The analyses were stratified by sex and controlled for age and menarche. Partial correlation coefficients and analysis of covariance were also analyzed.

Results In female students, ROM levels increased with increasing BMI and PBF. Therefore, ROM levels were significantly higher in the underweight group than in the BMI-classified overweight-obese $(P<0.001)$ and normal weight groups $(P<0.05)$. ROM levels were significantly higher in the high PBF group than in the underweight $(P<0.05)$ and normal groups $(P<0.001)$.

Conclusion The results of this study show that, regardless of menarche, obesity indicators such as BMI and PBF are
\end{abstract}

\section{T. Kogawa $(\bowtie)$}

Department of Health Promotion, Hirosaki University Graduate School of Health Sciences, 66-1 Hon-cho, Hirosaki, Aomori 036-8564, Japan

e-mail: t_kogawa@cc.hirosaki-u.ac.jp

\section{Kashiwakura}

Department of Radiological Life Sciences, Hirosaki University Graduate School of Health Sciences, 66-1 Hon-cho,

Hirosaki 036-8564, Japan correlated with the level of oxidative stress in female adolescents.

Keywords Oxidative stress $\cdot$ Obesity $\cdot$ Sex $\cdot$ Healthy adolescent

\section{Introduction}

In humans, oxidative stress is thought to be involved in several important pathways, potentially leading to the development of atherosclerosis [1, 2], endothelial dysfunction [3], and diabetes [4]. Lifestyle-related diseases have been reported to progress slowly from childhood [5] and are associated with high rates of occurrence and mortality in adults [6, 7]. A few cross-sectional studies have reported that oxidative stress and obesity are closely related [8-11], demonstrating a significant positive relationship between oxidative stress and obesity-related indices such as the body mass index (BMI, $\mathrm{kg} / \mathrm{m}^{2}$ ). In obese individuals, elevated levels of fatty acids increase the level of oxidative stress via activation of NADPH oxidase and increased production of reactive oxygen species (ROS) [10]. Obesity is generally regarded as a hyper-oxidative and chronic inflammatory state. The significant interrelationships among obesity-related indices, oxidative stress, inflammatory markers, and adipocytes have been well documented [9, 10, 12-14].

Obese children often develop into adults who are overweight or obese [15]. To prevent lifestyle-related diseases in adulthood, it is necessary to control adolescent obesity. However, the risk factors for obesity in children need to be identified before appropriate countermeasures against obesity can be developed, which in turn requires prior understanding of the oxidative stress in children. 
Previous studies on the relationship between oxidative stress and obesity have mainly focused on adults [7, 16$18]$, with attention paid to oxidative stress in adolescents [19]. The relationship between oxidative stress and obesity in healthy adolescents is also unknown. Therefore, a detailed, age-related understanding of oxidative stress is necessary.

To obtain useful insights for developing protective measures against lifestyle-related diseases, in this study we have measured redox levels in the blood using commercially available kits-the reactive oxygen metabolites (ROMs) test (i.e., the d-ROMs test) and the biological antioxidant potential (BAP) test, which have been found in previous studies to be useful indicators of oxidative stress [20-22].

\section{Materials and methods}

Study design

This study was performed in a rural area of Japan. Since 2006, medical health examinations, including peripheral blood collection, have been performed in these areas as a part of each town's "preventive medical health examination against lifestyle-related disease in young people." In our study, we surveyed 615 students (aged 12-15 years) in four junior high schools between April and May 2010. Before collecting the blood samples, we explained the purpose of the study, and an oral and written declaration was provided to specify that there would be no negative consequences for either the student or parent who did not wish to participate in the study. Students provided written assent and parents gave written consent for participation in this study, following which peripheral blood was collected from 595 students, yielding a participation rate of $96.7 \%$. There were 312 male students, ranging in age from 12 years 1 month to 15 years 0 months and 283 female students. This study was approved by the Committee of Medical Ethics of Hirosaki University Graduate School of Medicine (Hirosaki, Japan).

Measurement of obesity

In this study, BMI and percentage of body fat (PBF) were used as indicators of obesity [23]. BMI was calculated as weight in kilograms divided by the square of height in meters $\left(\mathrm{kg} / \mathrm{m}^{2}\right)$. Participants were classified as being of normal weight, overweight, or obese, based on their BMI, using the International Obesity Task Force classification [24]. These cutoff points are based on health-related adult definitions of overweight $\left(\geq 25 \mathrm{~kg} / \mathrm{m}^{2}\right)$ and obesity
( $\geq 30 \mathrm{~kg} / \mathrm{m}^{2}$ ), but they are adjusted according to age and sex of the study population.

Body weight and PBF were measured using an MC-190EM body composition analyzer (Tanita, Tokyo, Japan). These measurements were obtained while the participants were bare-footed and clad only in their underwear. The MC-190EM analyzer uses data acquired by dual X-ray absorptiometry (DXA) from both Japanese and Western subjects, as well as a regression formula derived from repeated regression analysis of height, weight, age, and impedance between the right hand and foot as variables. Criteria for childhood obesity were based on body fat percentage DXA standards for each sex and on the committee-created, childhood obesity manual from the Japan Society for the Study of Obesity. All students were classified into five groups according to their PBF: group 1, poor; group 2, (-)normal; group 3, (+)normal; group 4, slightly obese; group 5, obese.

Measurement of reactive oxygen metabolites

Reactive oxygen metabolites were measured using the d-ROMs test (Diacron International, Grosseto, Italy) which is a photometric test used to measure the concentration of hydroperoxides $(\mathrm{ROOH})$ in biological samples based on the principle of Fenton's reaction. Previous studies have shown $\mathrm{ROOH}$ to be a plasma biomarker predictive of a first atherothrombotic event [25]. The presence of $\mathrm{ROOH}$ in cells indicates an oxidative attack by ROS on various organic substrates, such as carbohydrates, lipids, amino acids, proteins, or nucleotides. In healthy subjects, the normal range of ROM is 250-300 Carratelli units (U.CARR) [26]. ROM was measured by using an automatic biochemical analyzer (TBA-120FR; Toshiba Medical Systems, Tokyo, Japan).

The BAP level was measured using the BAP test (Diacron International), which determines the biological antioxidant potential of blood plasma by measuring its ferric reducing ability, i.e., the transformation of iron from the ferric to the ferrous form. When the salt of a trivalent iron $\left(\mathrm{FeCl}_{3}\right)$ is dissolved in a colorless solution containing a chelation acid derivative, the solution turns red because of the action of the $\mathrm{Fe}^{3+}$ ions. This red solution is then decolorized by the addition of blood plasma due to the reduction of $\mathrm{Fe}^{2+}$ ions. In theory, the antioxidant potential of blood plasma can be evaluated by measuring the degree of decolorization using a photometer. The normal level of BAP in healthy subjects is $>2,200 \mu \mathrm{mol} / \mathrm{L}$. A value of $<2,200 \mu \mathrm{mol} / \mathrm{L}$ indicates a reduced BAP, indicating that the subject warrants clinical attention [27]. BAP was measured using a TBA-120FR automatic biochemical analyzer (Toshiba Medical Systems). 
In addition to the above measurements, we also performed a standard blood test using an XT-2000i Hematology Analyzer (Sysmex, Hyogo, Japan). This test measured white blood cell (WBC) counts, red blood cell (RBC) counts, platelet counts, hemoglobin levels, and hematocrit. The levels of aspartate aminotransferase (AST), alanine aminotransferase (ALT), total cholesterol (TC), triglyceride, high-density lipoprotein (HDL)-cholesterol, and low-density lipoprotein (LDL)-cholesterol were also analyzed using a TBA-120FR Chemistry Analyzer (Toshiba Medical Systems).

\section{Statistical analysis}

The significance of the differences in mean values between two groups were analyzed using Student's $t$ test. Data from the three obesity groups were analyzed using a one-way analysis of variance (ANOVA), with post hoc comparisons using the Tukey-Kramer multiple test. Multivariate linear regression analyses were associated with d-ROM level. The partial correlation coefficients and analysis of covariance (ANCOVA) were also analyzed. The analyses were stratified by sex and were controlled for age and menarche. Statistical analyses were performed using SPSS ver. 19.0 for Windows software (SPSS Japan, Tokyo, Japan). A value of $P<0.05$ was considered statistically significant.

\section{Results}

A total of 595 adolescents participated in the study. The incidence of obesity varied according to sex. According to the International Obesity Task Force cutoff, among the male students, $16.0 \%(n=50)$ were overweight and $4.8 \%$ $(n=15)$ were obese. Among the female students, $13.8 \%$ $(n=39)$ were overweight and $11.3 \%(n=11)$ were obese. The classification of male students into five groups according to their PBF was as follows: poor (PBF $<6.5, n=14$, $4.5 \%)$; (-)normal $(6.5 \leq \mathrm{PBF} \geq 15.4, n=168,53.8 \%)$; (+)normal (15.5 $\leq \mathrm{PBF} \geq 24.4, n=87,27.9 \%)$; slightly obese $\quad(24.5 \leq \mathrm{PBF} \geq 29.4, \quad n=14, \quad 14.5 \%)$; obese ( $\mathrm{PBF}>29.5, n=29,9.3 \%)$. The classification of female students into the five PBF groups was: poor $(\mathrm{PBF}<13.4$, $n=5,1.8 \%)$; (-)normal $(13.5 \leq \mathrm{PBF} \geq 22.4, n=103$,
Table 1 Characteristics of the subjects included in this study

$d$-ROMs Reactive oxygen metabolite (ROM) test, U.CARR Carratelli units

A $t$ test was used to compare parameters between the sexes

$* P<0.05$, ** $P<0.01$,

*** $P<0.001$

\begin{tabular}{|c|c|c|c|c|c|c|c|}
\hline \multirow[t]{2}{*}{ Items } & \multicolumn{2}{|c|}{ All $(n=595)$} & \multicolumn{2}{|c|}{ Male $(n=312)$} & \multicolumn{2}{|c|}{ Female $(n=283)$} & \multirow[t]{2}{*}{$P$} \\
\hline & Mean & $\begin{array}{l}\text { Standard } \\
\text { deviation }\end{array}$ & Mean & $\begin{array}{l}\text { Standard } \\
\text { deviation }\end{array}$ & Mean & $\begin{array}{l}\text { Standard } \\
\text { deviation }\end{array}$ & \\
\hline Height $(\mathrm{cm})$ & 157.1 & 8.2 & 159.4 & 9.1 & 154.6 & 6.2 & ${ }^{* * *}$ \\
\hline Weight (kg) & 49.9 & 10.3 & 51.0 & 10.8 & 48.7 & 9.6 & ${ }^{* *}$ \\
\hline Body mass index $\left(\mathrm{kg} / \mathrm{m}^{2}\right)$ & 20.1 & 3.2 & 19.9 & 3.1 & 20.3 & 3.4 & \\
\hline Percentage body fat & 20.5 & 9.0 & 16.2 & 8.2 & 25.2 & 7.3 & ${ }^{* * * *}$ \\
\hline Body fat (kg) & 10.8 & 6.7 & 8.9 & 6.4 & 12.9 & 6.4 & ${ }^{* * * *}$ \\
\hline $\begin{array}{l}\text { Systolic blood pressure (BP) } \\
(\mathrm{mmHg})\end{array}$ & 110.5 & 8.4 & 111 & 8.5 & 109 & 8.1 & $* *$ \\
\hline Diastolic BP (mmHg) & 63.0 & 8.7 & 62 & 9.1 & 64 & 8.2 & \\
\hline $\begin{array}{l}\text { Aspartate aminotransferase } \\
\text { (U/L) }\end{array}$ & 21.1 & 5.2 & 23.1 & 5.7 & 18.9 & 3.4 & **** \\
\hline $\begin{array}{l}\text { Alanine aminotransferase } \\
(\mathrm{mg} / \mathrm{dL})\end{array}$ & 14.9 & 9.3 & 17.0 & 11.7 & 12.5 & 4.7 & ${ }^{* * * *}$ \\
\hline Total cholesterol (mg/dL) & 162.1 & 25.6 & 157.6 & 26.4 & 167.0 & 23.7 & ${ }^{* * * *}$ \\
\hline Triglycerides (mg/dL) & 73.9 & 42.4 & 70.5 & 44.4 & 77.7 & 39.8 & $*$ \\
\hline $\begin{array}{l}\text { High-density lipoprotein- } \\
\text { cholesterol (mg/dL) }\end{array}$ & 59.4 & 14.4 & 58.7 & 14.8 & 60.0 & 13.9 & \\
\hline $\begin{array}{l}\text { Low-density lipoprotein- } \\
\text { cholesterol (mg/dL) }\end{array}$ & 83.6 & 21.2 & 80.2 & 21.8 & 87.3 & 19.9 & **** \\
\hline White blood cells $\left(\times 10^{3} / \mu \mathrm{L}\right)$ & 61.3 & 13.9 & 59.3 & 12.9 & 63.5 & 14.6 & *** \\
\hline Red blood cells $\left(\times 10^{4} / \mu \mathrm{L}\right)$ & 486.1 & 38.4 & 503.6 & 35.1 & 466.7 & 32.1 & ${ }^{* * *}$ \\
\hline Hemoglobin (g/dL) & 13.5 & 1.3 & 14.0 & 1.2 & 13.0 & 1.2 & ${ }^{* * * *}$ \\
\hline Hematocrit (\%) & 42.1 & 3.5 & 43.2 & 3.4 & 40.9 & 3.1 & ${ }^{* * *}$ \\
\hline Platelets $\left(\times 10^{4} / \mu \mathrm{L}\right)$ & 26.9 & 5.4 & 26.9 & 5.2 & 26.9 & 5.6 & \\
\hline d-ROMs (U.CARR) & 304.0 & 59.2 & 299.9 & 55.2 & 308.6 & 63.1 & \\
\hline $\begin{array}{l}\text { Biological antioxidant } \\
\text { potential }(\mu \mathrm{m} / \mathrm{L})\end{array}$ & 2713.9 & 119.8 & 2745.5 & 108.1 & 2679.0 & 122.6 & $* * * *$ \\
\hline
\end{tabular}


Fig. 1 Correlations between body mass index $(B M I)$, percentage body fat $(P B F)$, and reactive oxygen metabolite (ROM) test ( $d$-ROMs) levels in all male and female students. A significantly positive correlation was observed for each combination
Fig. 2 Correlations between BMI, PBF, and biological antioxidant potential $(B A P)$ levels in all male and female students. No correlations were observed
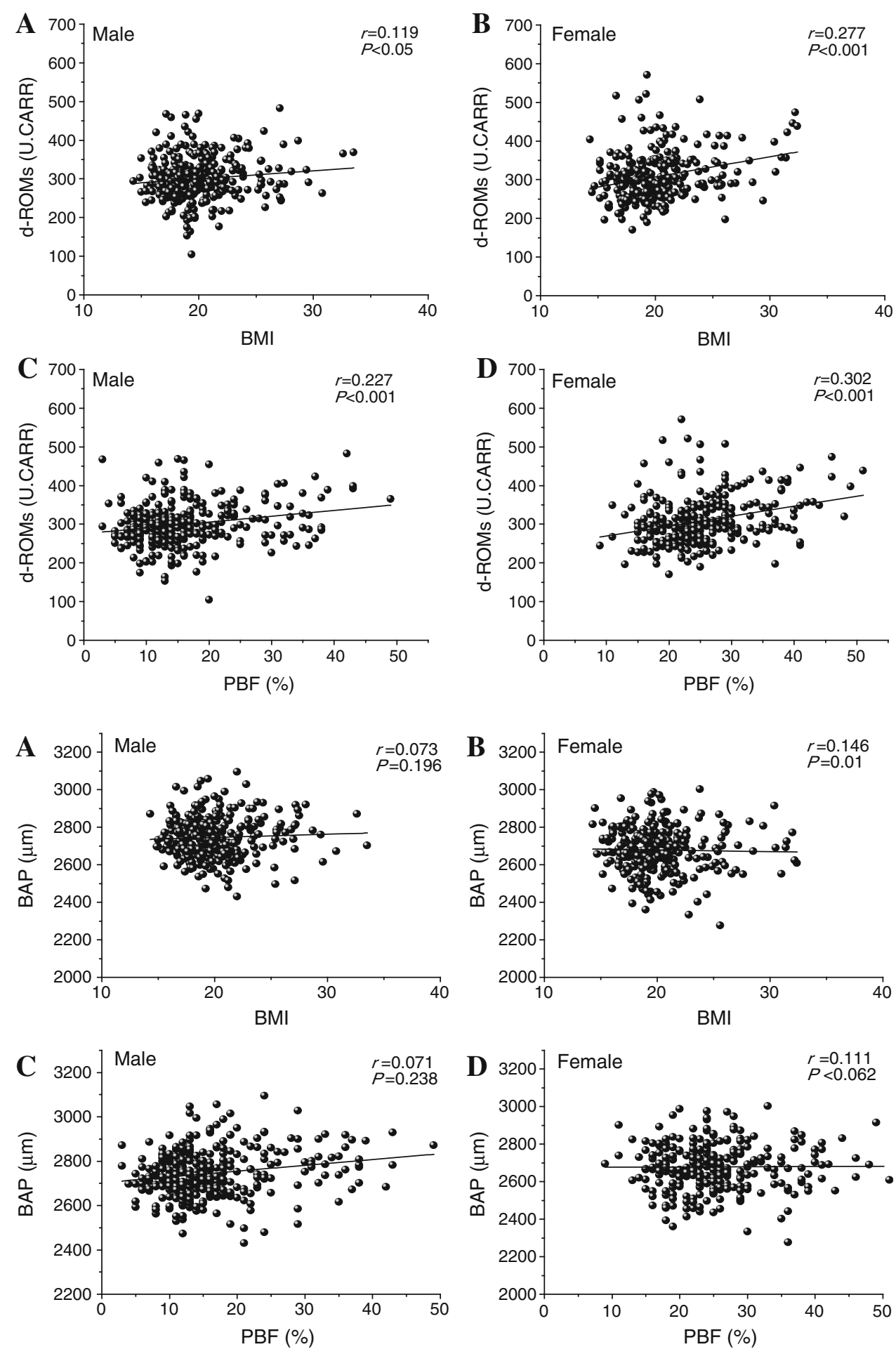

$36.4 \%)$; (+)normal $(22.5 \leq \mathrm{PBF} \geq 31.4, n=128,45.2 \%)$; slightly obese $(31.5 \leq \mathrm{PBF} \geq 35.4, n=15,5.3 \%)$; obese (PBF $>35.5, n=32,11.3 \%)$.

Table 1 summarizes the mean value of each parameter, such as height, weight, BMI, PBF, systolic blood pressure (BP), diastolic BP, AST, ALT, TC, triglyceride, HDLcholesterol, LDL-cholesterol, WBC, RBC, and platelet counts, hemoglobin, hematocrit, d-ROMs, and BAP.
The values for body fat, PBF, TC, LDL-cholesterol, and WBCs were significantly higher in female students than in male students $(P=0.001)$, while the values for height, AST, ALT, RBCs, hemoglobin, hematocrit, and BAP were significantly higher in male students than in female students $(P=0.001)$. The mean level of $\mathrm{d}$-ROMs was $304 \pm 59.2$ (U. CARR), with no statistically significant difference between the sexes. 
Fig. 3 Comparison of serum d-ROMs levels detected in the three BMI groups and in each PBF level group in male and female students. a In male students, a significant difference was not observed between serum d-ROMs levels and BMI, b in female students, d-ROMs levels were higher in females with a BMI of $<18.5$ than in other females, $\mathbf{c}$ in male students, a significant difference was not observed between serum d-ROMs levels and PBF grades, $\mathbf{d}$ in female students, the d-ROMs levels in group 5 females were significantly higher than those in group 2 and group 3 females. $* P<0.05$, $* * P<0.01, * * * P<0.001$. PBF grades $(1-5, x$-axis): 1 poor, $2(-)$ normal, 3 (+)normal, 4 slightly obese, 5 obese
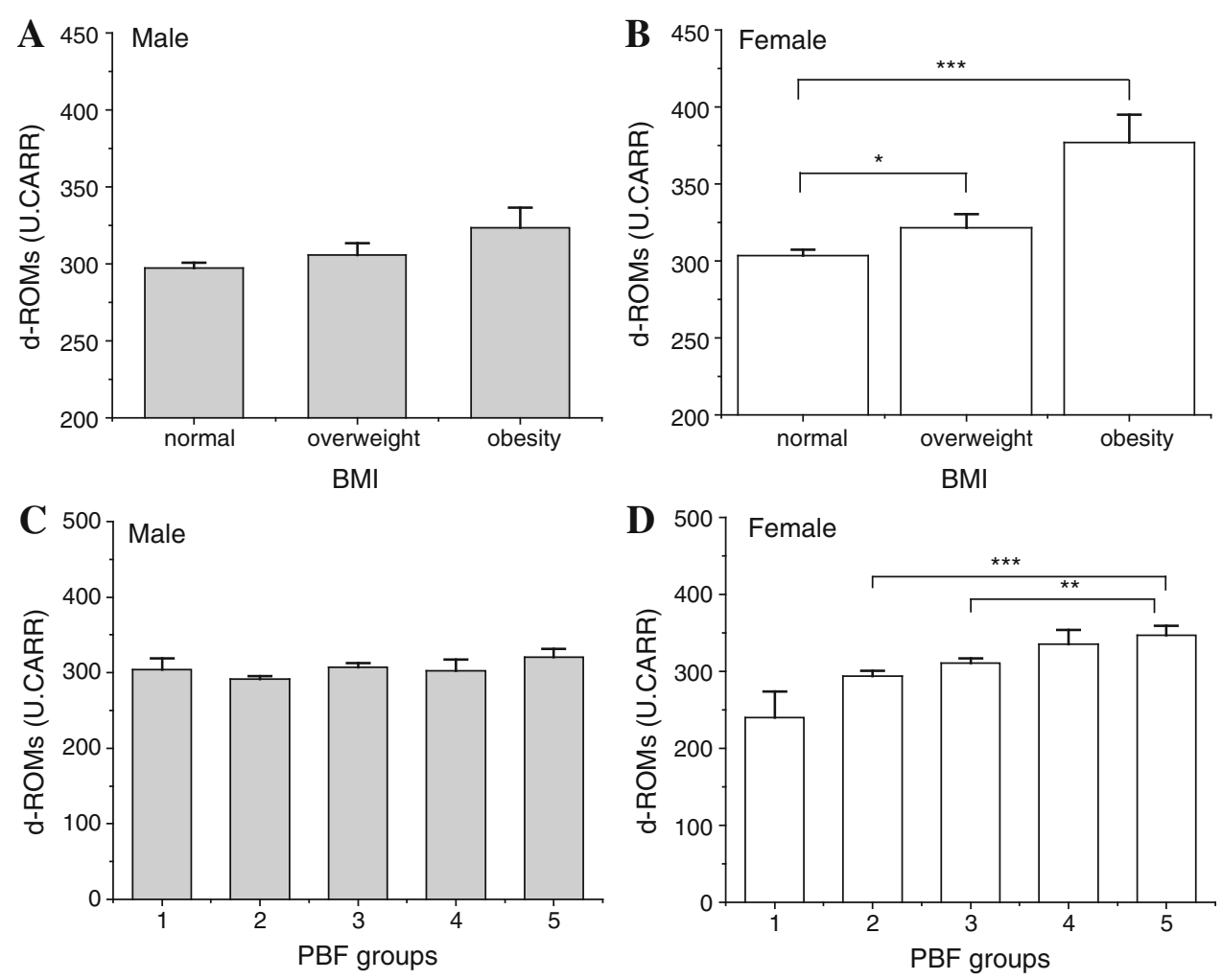

The correlations between d-ROMs and BMI or PBF were analyzed (Fig. 1). Both BMI (male students, $r=0.12$; female students, $r=0.277$; all $P<0.001)$ and PBF (male students, $r=0.23$; female students, $r=0.30$; all $P<0.001)$ were positively correlated to d-ROMs in both the sexes. However, BAP was not correlated with BMI (male students, $r=0.07$; female students, $r=0.07$ ) or PBF (male students, $r=0.15$; female students, $r=0.11$ ) (Fig. 2).

To determine the correlation between d-ROMs and BMI levels, each student was classified into one of three groups based on his/her BMI level, as described in the "Materials and methods". The d-ROMs levels were observed to increase depending on the BMI group among the female students. However, no significant difference was observed among the three groups of male students (Fig. 3). In the case of PBF, similar results were also observed among female students, but not among males. The d-ROMs levels in obese females were significantly higher than those in the $(-)$ normal and $(+)$ normal groups. The incidence of obesity and the correlation between d-ROMs and the obesity indices varied according to sex.

\section{Discussion}

The present study was performed to clarify the relationship between obesity and serum d-ROMs or BAP levels in adolescents. Our findings show that the levels of d-ROMs were correlated with obesity levels to some extent (Fig. 1), with a clear correlation being observed among female students (Fig. 2). A similar tendency was also observed in a previous adult study [9]; however, few studies have reported on this correlation in adolescents. Mehdad et al. [28] showed that fasting blood glucose levels are highly correlated with fat mass and PBF in overweight-obese female adolescents aged 11-17 years, a population similar in age to our study sample. Cruz et al. [29] reported that this positive relationship in the overweight-obese group of girls may be explained by the clustering of metabolic syndrome factors, which are risk factors for type 2 diabetes. Regarding ROS production, Furukawa et al. [10] suggested that increased oxidative stress in the plasma is due to increased ROS production from accumulated fat. In addition, Fernández-Sánchez et al. [30] reported that white adipose tissue may produce certain bioactive substances called adipokines, which induce the production of ROS. In our study, female students showed significantly higher fat-related or obesity-related factors, such as PBF, body fat, TC, triglycerides, and LDL-cholesterol, than did male students (Table 1).

Although there are sex-based differences in sex hormones and body composition among adolescents, these findings suggest the possibility that d-ROMs levels are physiologically related to ROS produced by the fat cells. 
In other words, the levels of d-ROMs, especially in female students, may be a biological marker of obesity in adolescence. On the other hand, the mean value of BAP in male students was significantly higher than that in female students, as shown in Table 1. However, BAP levels were not correlated with obesity in male students (Fig. 3). Although the precise mechanisms are not yet clear, some of the factor(s) discussed above, except for fat-related or obesity-related factors, may be involved. Further investigation of these mechanisms is warranted to clarify this mechanism.

The subjects of this study were adolescent, junior high school students. The development of secondary sex characteristics is known to occur earlier in girls than in boys. Thus, it is possible that sex hormones play an important role in the correlation between the d-ROMs levels and obesity that was observed among female students. However, this was a cross-sectional study, and the data set was obtained in a specific region over a limited period. More precise approaches are currently underway to examine the effect of oxidative stress during these development stages.

Spalding et al. [31] reported that the number of fat cells remains constant in adulthood in lean and obese individuals, even after marked weight loss, indicating that the number of adipocytes is determined during childhood and adolescence. As Furukawa reported, this suggests that increased oxidative stress in accumulated fat is an early indication of metabolic syndrome and that the redox state in adipose tissue is a potentially useful therapeutic target for obesity-associated metabolic syndrome. Therefore, appropriate weight control in adolescence may help avoid metabolic syndrome in adulthood.

Furthermore, our study focused on student's obesity in relation to their serum ROM. The low correlation coefficient between d-ROMs levels and obesity was not considered as a factor indicative of a subject's lifestyle; further analysis is required to account for differences between lifestyles. Moreover, prevention of lifestyle-related diseases requires follow-up health examinations. In particular, with the aim of preventing future lifestyle-related diseases, health education would be especially valuable for the highrisk groups shown in Fig. 3 (obesity and PBF levels 3, 4, and 5), who exhibited a high level of oxidative stress. Counseling with regard to healthy eating habits can be recommended to improve the lifestyle of these high-risk individuals. Moreover, this type of lifestyle education would be most effective if it involved both junior high school students and their guardians. Further studies, such as a prospective cohort study, would be required to confirm the transformation of the high-risk factors associated with this group.

In conclusion, the results of our study reveal the presence of a relationship between oxidative stress and obesity among adolescents in rural Japan. Since there is a possibility that adolescent obesity increases the risk of various diseases in adulthood, we believe that an estimation of obesity, based on d-ROMs, is very important for female teenagers.

Acknowledgments This study was supported, in part, by the Health \& Welfare Section of Nanbu Town, teachers at the junior high school of Nanbu, and the National Health Insurance Nagawa Hospital, Japan. This work was partly supported by a Grant-in-Aid for Scientific Research (C) of the Japan Society for the Promotion of Science (No. 21592845, TK).

Conflict of interest None.

\section{References}

1. Emdin M, Pompella A, Paolicchi A. Gamma-glutamyltransferase, atherosclerosis, and cardiovascular disease: triggering oxidative stress within the plaque. Circulation. 2005;112:2078-80.

2. Itoh S, Umemoto S, Hiromoto M, Toma Y, Tomochika Y, Aoyagi $\mathrm{S}$, et al. Importance of NAD (P) H oxidase-mediated oxidative stress and contractile type smooth muscle myosin heavy chain SM2 at the early stage of atherosclerosis. Circulation. 2002;105:2288-95.

3. Perticone F, Ceravolo R, Candigliota M, Ventura G, Iacopino S, Sinopoli F, et al. Obesity and body fat distribution induce endothelial dysfunction by oxidative stress: protective effect of vitamin C. Diabetes. 2001;50:159-65.

4. Robertson RP. Chronic oxidative stress as a central mechanism for glucose toxicity in pancreatic islet beta cells in diabetes. J Biol Chem. 2004;279:42351-4.

5. Seidell JC, Flegal KM. Assessing obesity: classification and epidemiology. Br Med Bull. 1997;53:238-52.

6. Griendling KK, FitzGerald GA. Oxidative stress and cardiovascular injury: part II: animal and human studies. Circulation. 2003;108:2034-40.

7. Lacy F, O'Connor DT, Schmid-Schönbein GW. Plasma hydrogen peroxide production in hypertensives and normotensive subjects at genetic risk of hypertension. J Hypertens. 1998;16:291-303.

8. Atabek ME, Vatansev H, Erkul I. Oxidative stress in childhood obesity. J Pediatr Endocrinol Metab. 2004;17:1063-8.

9. Keaney JF Jr, Larson MG, Vasan RS, Wilson PW, Lipinska I, Corey D, et al. Obesity and systemic oxidative stress: clinical correlates of oxidative stress in the Framingham Study. Arterioscler Thromb Vasc Biol. 2003;23:434-9.

10. Furukawa S, Fujita T, Shimabukuro M, Iwaki M, Yamada Y, Nakajima $\mathrm{Y}$, et al. Increased oxidative stress in obesity and its impact on metabolic syndrome. J Clin Invest. 2004;114:1752-61.

11. Urakawa H, Katsuki A, Sumida Y, Gabazza EC, Murashima S, Morioka $\mathrm{K}$, et al. Oxidative stress is associated with adiposity and insulin resistance in men. J Clin Endocrinol Metab. 2003;88: 4673-6.

12. Nakanishi S, Yamane K, Kamei N, Nojima H, Okubo M, Kohno $\mathrm{N}$. A protective effect of adiponectin against oxidative stress in Japanese Americans: the association between adiponectin or leptin and urinary isoprostane. Metabolism. 2005;54:194-9.

13. Shin MJ, Lee JH, Jang Y, Park E, Oh J, Chung JH, et al. Insulin resistance, adipokines, and oxidative stress in nondiabetic, hypercholesterolemic patients: leptin as an 8-epi-prostaglandin F2alpha determinant. Metabolism. 2006;55:918-22. 
14. Davi G, Guagnano MT, Ciabattoni G, Basili S, Falco A, Marinopiccoli M, et al. Platelet activation in obese women: role of inflammation and oxidant stress. JAMA. 2002;288:2008-14.

15. Togashi K, Masuda H, Rankinen T, Tanaka S, Bouchard C, Kamiya H. A 12-year follow-up study of treated obese children in Japan. Int J Obes Relat Metab Disord. 2002;26(6):770-7.

16. Belch JJ, Bridges AB, Scott N, Chopra M. Oxygen free radicals and congestive heart failure. Br Heart J. 1991;65:245-8.

17. Mallat Z, Philip I, Lebret M, Chatel D, Maclouf J, Tedgui A. Elevated levels of 8-iso-prostaglandin F2 $\alpha$ in pericardial fluid of patients with heart failure. Circulation. 1998;97:1536-9.

18. Vaziri ND. Roles of oxidative stress and antioxidant therapy in chronic kidney disease and hypertension. Curr Opin Nephrol Hypertens. 2004;13:93-9.

19. Kogawa T, Nishimura M, Kurauchi S, Kashiwakura I. Characteristics of reactive oxygen metabolites in serum of early teenagers in Japan. Environ Health Prev Med. 2012;17:364-70.

20. Buonocore G, Perrone S, Longini M, Vezzosi P, Marzocchi B, Paffetti $\mathrm{P}$, et al. Oxidative stress in preterm neonates at birth and on the seventh day of life. Pediatr Res. 2002;52:46-9.

21. Ippolito S, Ambroso G, Mani AR, Novembrino C, Lonati S, Ponticelli $\mathrm{C}$, et al. Oxidative and nitrative status in preeclampsia and normal pregnancy (abstract). Clin Chem Lab Med. 2003;41[Special Suppl]:301.

22. Wisdom SJ, Wilson R, McKillop JH, Walker JJ. Antioxidant systems in normal pregnancy and in pregnancy-induced hypertension. Am J Obstet Gynecol. 1991;165:1701-4.

23. Rosner B, Prineas R, Loggie J, Daniels SR. Percentiles for body mass index in U.S. children 5 to 17 years of age. J Pediatr. 1998;132:211-22.
24. Cole TJ, Bellizzi MC, Flegal KM, Dietz WD. Establishing a standard definition for child overweight and obesity worldwide: international survey. BMJ. 2000;320:1240-3.

25. Alberti A, Bolognini L, Macciantelli D, Caratelli M. The radical cation of N, N-diethyl-para-phenylendiamine: a possible indicator of oxidative stress in biological samples. Res Chem Intermediates. 2000;26:253-67.

26. Benzie F, Strain JJ. The ferric reducing ability of plasma (FRAP) as a measure of "Antioxidant Power": the FRAP assay. Anal Biochem. 1996;239:70-6.

27. Belcaro G, Carratelli M, Cesarone MR, Cornelli U, Incandela L, Nicolaides A. Oxygen free radicals: a method, a product, an application (abstract). In: Proc SFRR Europe Summer Meeting. Abano Terme, Padua; 1997;183-184.

28. Mehdad S, Hamrani A, El Kari K, et al. Body mass index, waist circumference, body fat, fasting blood glucose in a sample of moroccan adolescents aged 11-17 years. J Nutr Metab. 2012; 2012:510458. doi:10.1155/2012/510458.

29. Cruz ML, Goran MI. The metabolic syndrome in children and adolescents. Curr Diab Rep. 2004;4:53-62.

30. Fernández-Sánchez A, Madrigal-Santillán E, Bautista M, Esquivel-Soto J, Morales-González A, Esquivel-Chirino C, et al. Inflammation, oxidative stress, and obesity. Int J Mol Sci. 2011; 12(5):3117-32.

31. Spalding KL, Arner E, Westermark PO, Bernard S, Buchholz BA, Bergmann $\mathrm{O}$, et al. Dynamics of fat cell turnover in humans. Nature. 2008;453:783-7. 